УДК 004.85

\title{
СРАВНИТЕЛЬНЫЙ АНАЛИЗ МЕТОДОВ МАШИННОГО ОБУЧЕНИЯ ДЛЯ РЕШЕНИЯ ЗАДАЧИ КЛАССИФИКАЦИИ В СРЕДЕ WOLFRAM MATHEMATICA
}

\author{
Хлебородова Лидия Дмитриевна \\ студент \\ Научный руководитель: Осипов Геннадий Сергеевич \\ д.т.н., зав. кафедрой Информатики \\ ФГБОУ ВО «Сахалинский государственный университет»
}

\begin{abstract}
Аннотация: Апробирована унифицированная методология решения задачи классификации сложных объектов на больших данных методами машинного обучения в среде Wolfram Mathematica. Проведен сравнительный анализ точности классификации 9 методами на примере базы данных по автомобилям, содержащей как числовую, так и символьную информацию.

Ключевые слова: задача классификации, методы машинного обучения, компьютерный анализ.

\section{COMPARATIVE ANALYSIS OF MACHINE LEARNING METHODS FOR SOLVING THE CLASSIFICATION PROBLEM IN THE WOLFRAM MATHEMATICA ENVIRONMENT}

\section{Khleborodova Lidiia Dmitrievna Osipov Gennady Sergeevich}

\footnotetext{
Abstract: A unified methodology for solving the problem of classifying complex objects on big data using machine learning methods in the Wolfram Mathematica environment has been tested. A comparative analysis of the accuracy of classification by 9 methods is carried out on the example of a database on cars containing both numerical and symbolic information.

Key words: classification problem, machine learning methods, computer analysis.
} 


\section{Структура обучающей выборки}

На рисунке 1 представлен фрагмент обучающей выборки [1], в которой $m=1728$ результата наблюдений, $n=6$ входных переменных: $x=\left\{x_{1}, x_{2}, . ., x_{6}\right\}$ и одна выходная переменная $y$.

\begin{tabular}{|l|l|c|c|l|l|l|}
\hline \multicolumn{1}{|c|}{$\mathbf{x}_{\mathbf{1}}$} & \multicolumn{1}{c|}{$\mathbf{x}_{\mathbf{2}}$} & $\mathbf{x}_{\mathbf{3}}$ & $\mathbf{x}_{\mathbf{4}}$ & \multicolumn{1}{c|}{$\mathbf{x}_{\mathbf{5}}$} & \multicolumn{1}{c|}{$\mathbf{x}_{\mathbf{6}}$} & \multicolumn{1}{c|}{$\mathbf{y}$} \\
\hline vhigh & vhigh & 2 & 2 & small & low & unacc \\
\hline med & med & 2 & more & big & med & acc \\
\hline vhigh & vhigh & 2 & 2 & small & high & unacc \\
\hline vhigh & vhigh & 2 & 2 & med & low & unacc \\
\hline low & low & 5 more & more & big & low & unacc \\
\hline low & low & 5 more & more & big & med & good \\
\hline low & low & 5 more & more & big & high & vgood \\
\hline vhigh & vhigh & 2 & 2 & med & med & unacc \\
\hline
\end{tabular}

Рис. 1. Фрагмент обучающей выборки

\section{Постановка задачи}

Объектом и предметом исследования является проблема построения классификатора $f: x \rightarrow y$,

где $x=\left(x_{1}, x_{2}, . ., x_{6}\right)$ - вектор числовых и строковых данных;

$y$ - идентификатор класса, к которому относится объект.

Цель исследования - проведение сравнительного анализа применимости различных методов машинного обучения [2] для решения задачи классификации.

\section{Описание исходных данных}

Наименование входных данных $(x)$ :

$>$ Общая цена (total price):

1. цена покупки (purchase price);

2. стоимость обслуживания (cost of maintenance).

$>$ Техническте характерирстики (technical parameters):

3. количество дверей (number of doors);

4. вместимость (людей для перевозки) (capacity (people for transportation));

5. размер багажника (trunk size);

6. безопасность автомобиля (car safety). 
На рисунке 2 приведена иерархическая структура исходных данных

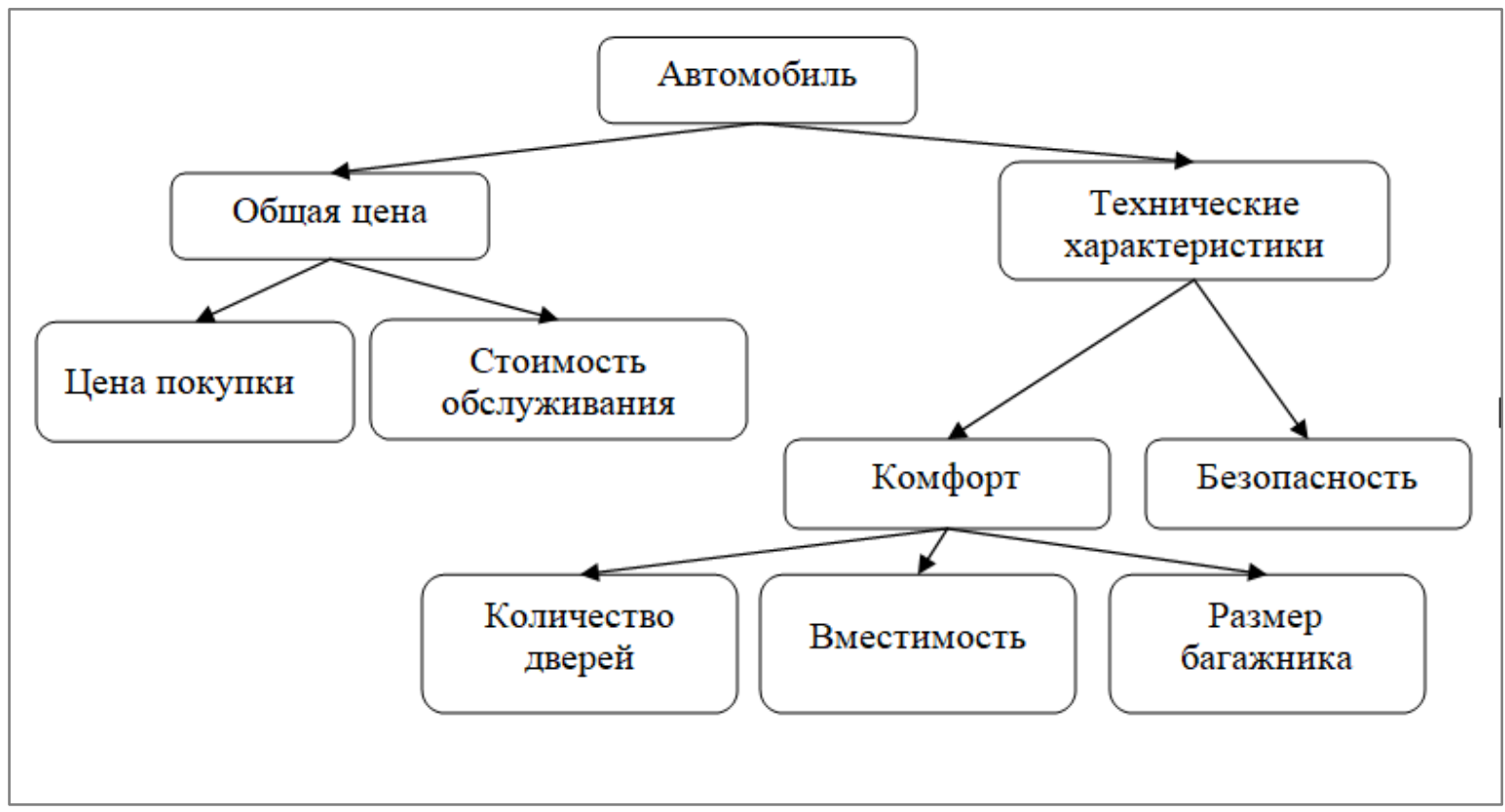

Рис. 2. Сетевая модель исходных данных

В таблице 1 приведены возможные значения для $n=6$ входных переменных и наименования четырех классов.

Таблица 1

Множество значений переменных

\begin{tabular}{|c|c|c|c|c|c|c|}
\hline$x_{1}$ & 2 & $x_{3}$ & $x_{4}$ & $x_{5}$ & $x_{6}$ & $y$ \\
\hline $\begin{array}{l}\text { vhigh, } \\
\text { high, } \\
\text { med, } \\
\text { low }\end{array}$ & $\begin{array}{l}\text { vhigh, } \\
\text { high, } \\
\text { med, } \\
\text { low }\end{array}$ & $\begin{array}{c}2, \\
3, \\
4, \\
5 \text { more }\end{array}$ & $\begin{array}{c}2, \\
4, \\
\text { more }\end{array}$ & $\begin{array}{c}\text { small, } \\
\text { med, } \\
\text { big }\end{array}$ & $\begin{array}{l}\text { low } \\
\text { mec } \\
\text { hig }\end{array}$ & $\begin{array}{c}\text { unace, } \\
\text { acc, } \\
\text { good, } \\
\text { vgood }\end{array}$ \\
\hline
\end{tabular}

\section{Методы исследования}

Для достижения цели исследования реализовывались следующие методы машинного обучения $[3,4,5]$ :

1. дерево решений (DecisionTree);

2. градиентный бустинг (GradientBoostedTrees;

3. логистическая регрессия (LogisticRegression);

4. модель Маркова (Markov);

5. наивный Байес (NaiveBayes);

6. ближайший сосед (NearestNeighbors); 
7. нейронная сеть (NeuralNetwork);

8. случайный лес (RandomForest);

9. опорные вектора (SupportVectorMachine).

Компьютерное моделирование и практическая апробация системы классификации на большом массиве разнородных данных выполнялись на базе аналитической платформы Wolfram Mathematica [6], имеющей в своем арсенале алгоритмы решения задач построения систем искусственного интеллекта.

\section{Основные результаты}

На рисунке 3 приведен оператор построения модели классификации, например, методом Логистической регрессии.

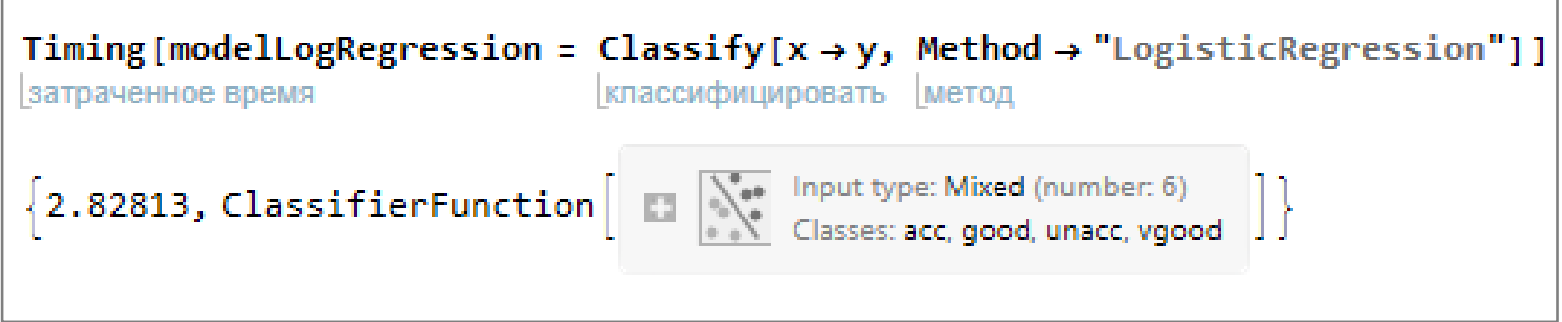

\section{Рис. 3. Обучение модели}

Подробная информация о параметрах процесса обучения приведена на рисунке 4.

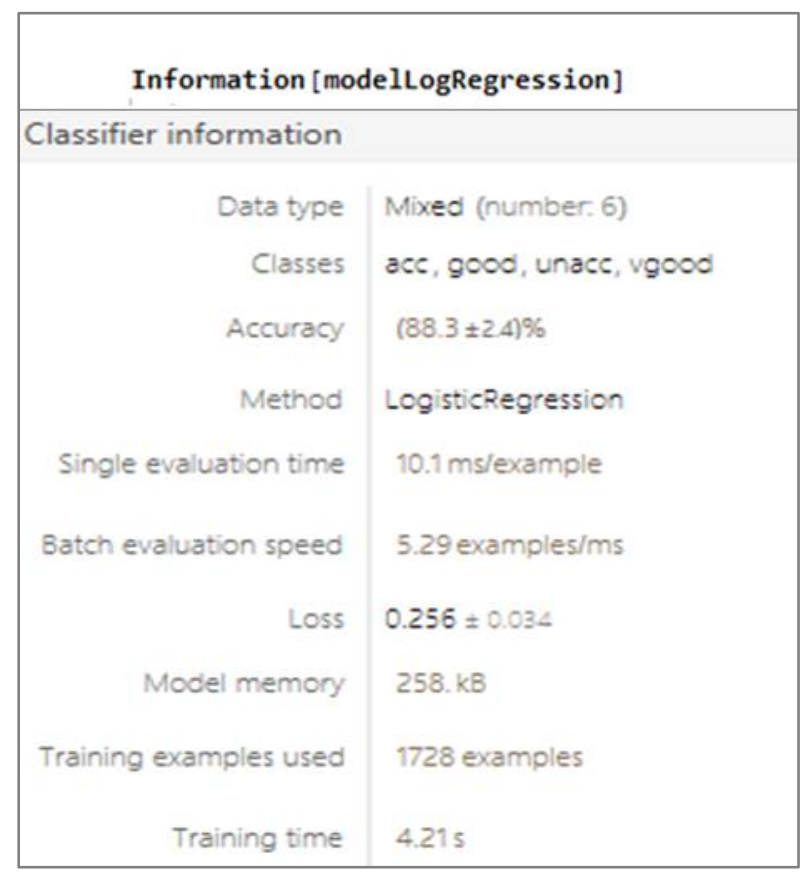

Рис. 4. Информация о процессе обучения 
Дополнительная графическая информация об изменении в процессе обучения точности классификации и кривая обучения на тестовом наборе данных приведены на рисунке 5.

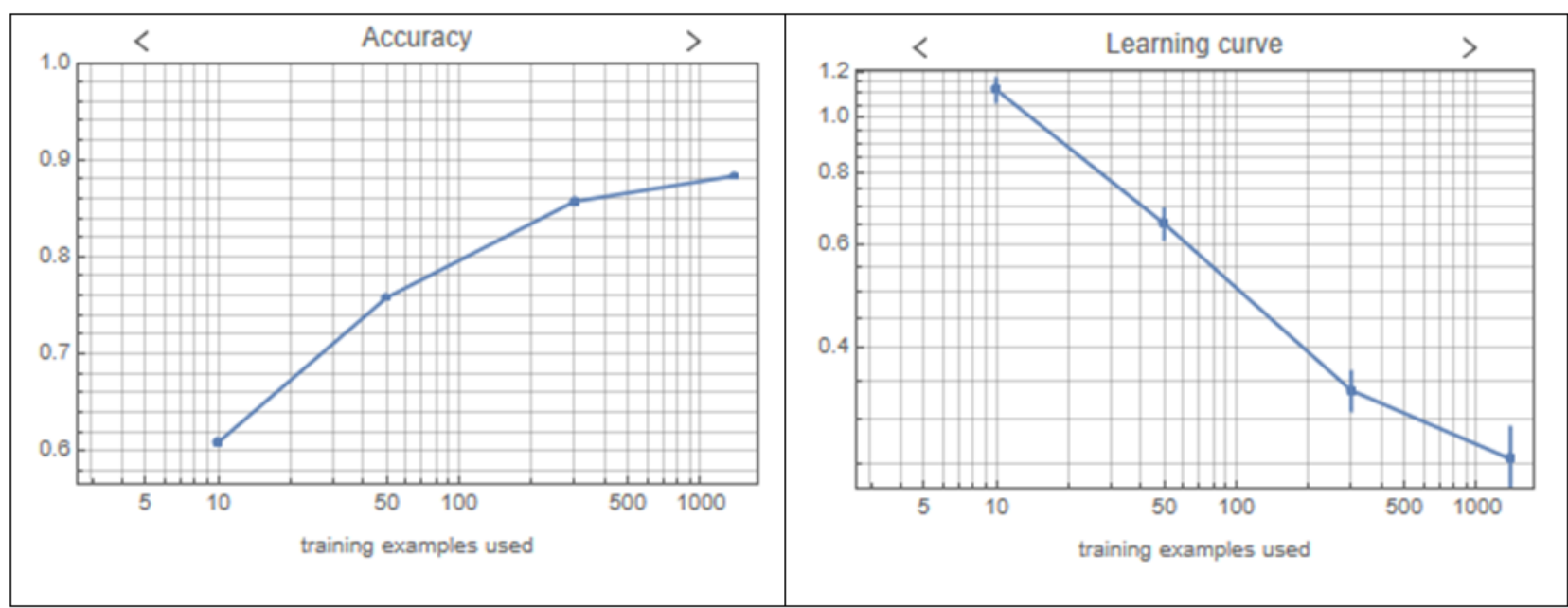

Рис. 5. Изменение точности классификации и кривая обучения

Операторы проверки системы классификации, обученной методом случайного леса, на тестовой выборке приведены на рисунке 6.

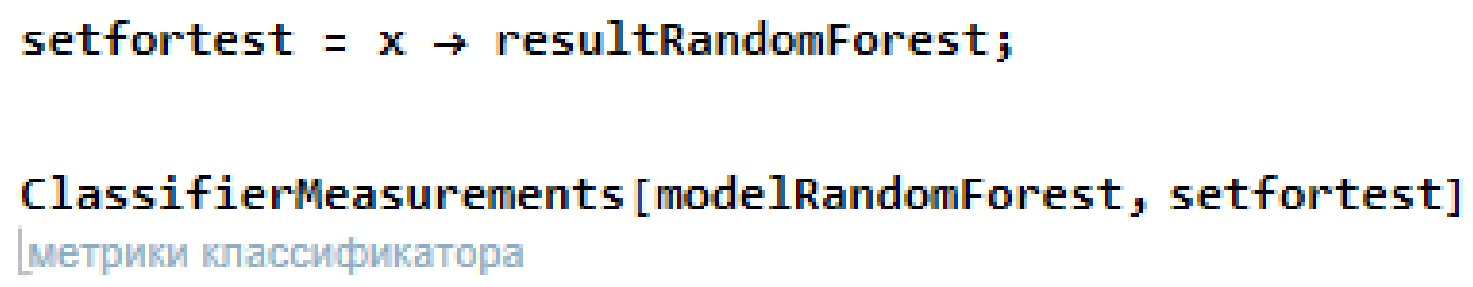

Рис. 6. Тестирование модели на обучающей выборке

На рисунке 7 даны сведения о процессе обучения системы классификации и матрица ошибок распознавания классов. 


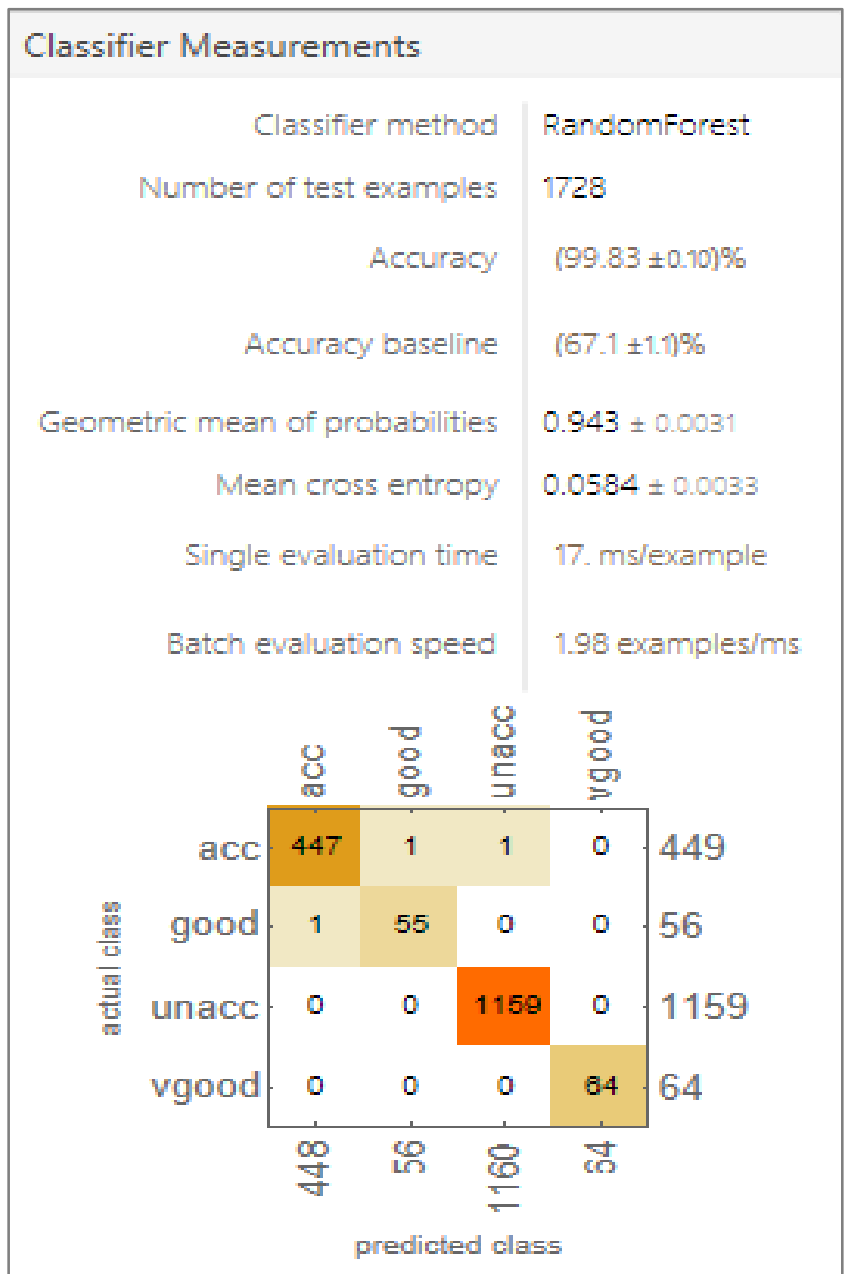

Рис. 7. Информация об ошибках классификации

Основные результаты исследования - показатели качества классификации различными методами представлены в таблице 2.

Таблица 2

Основные оценки качества классификации

\begin{tabular}{|c|c|c|}
\hline Название метода & Время на моделирование, с & Ошибка классификации, \% \\
\hline Дерево решений & 5.77 & 14.4 \\
\hline Градиентный бустинг & 3.78 & 5.3 \\
\hline Логистическая регрессия & 1.19 & 10.0 \\
\hline Модель Маркова & 0.52 & 10.8 \\
\hline Наивный Байес & 0.31 & 13.4 \\
\hline Ближайший сосед & 0.27 & 21.4 \\
\hline Нейронная сеть & 11.39 & 0.4 \\
\hline Случайный лес & 2.33 & 7.1 \\
\hline Опорные вектора & 8.36 & 10.1 \\
\hline
\end{tabular}


После нормирования экспериментальных данных и, учитывая преимущество критерия ошибки классификации над временем обучения модели (в соответствии со шкалой сравнений Саати), получим оценки степени предпочтительности методов машинного обучения применительно к задаче классификации, представленные на рисунке 8.

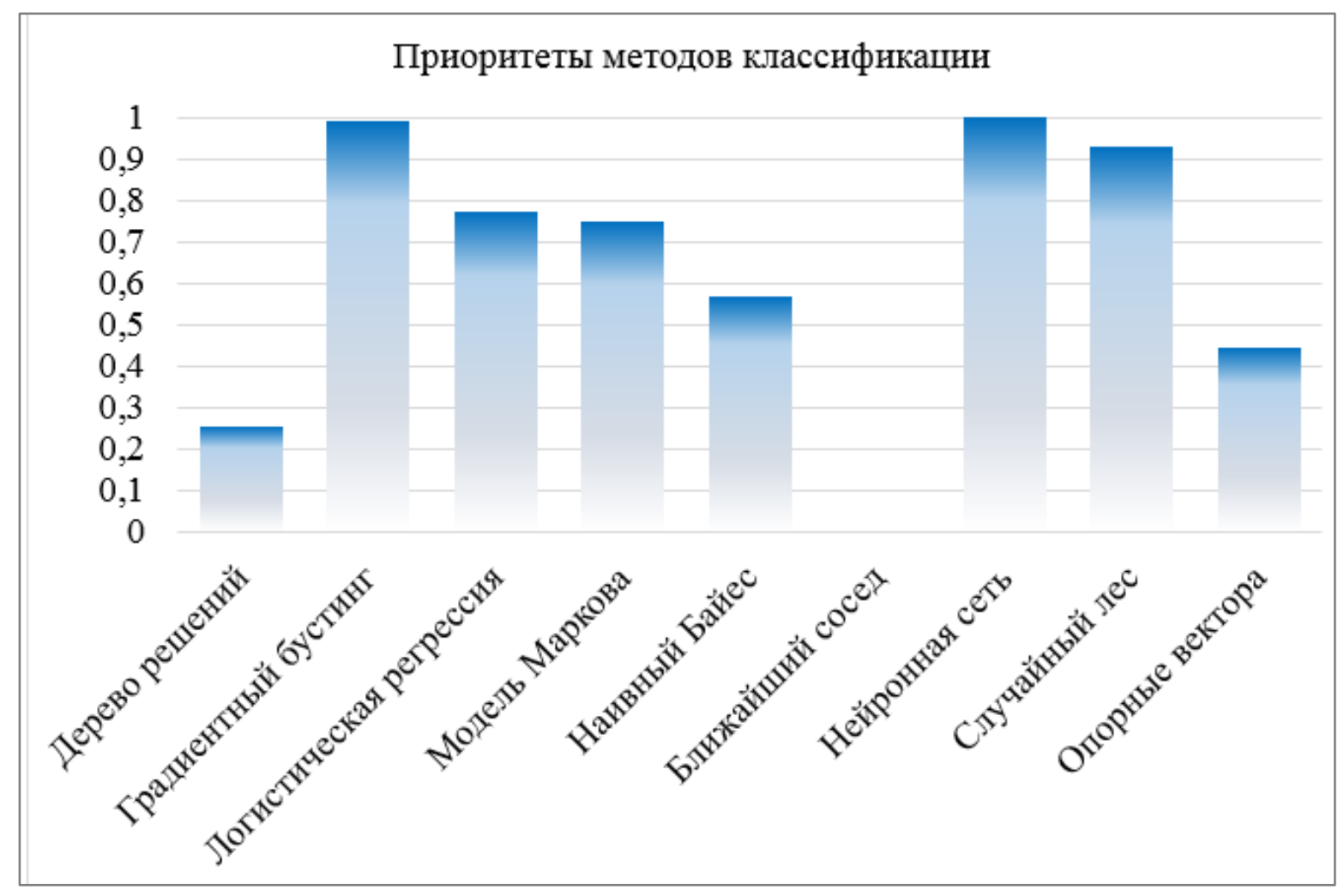

Рис. 8. Ранжирование методов машинного обучения

Из полученных данных следует, что наивысшим приоритеттом обладает метод классификации, основанный на использовании искусственной нейронной сети.

\section{Выводы}

Произведен сравнительный анализ 9 методов машинного обучения для решения задачи классификации автомобилей на примере обучающей выборки, состоящей из неоднородных данных (числовых и строковых) большой размерности. 


\section{Список литературы}

1. Car Evaluation Data Set.

2. URL: https://archive.ics.uci.edu/ml/datasets/car+evaluation.

3. А. Дьяконов «Введение в анализ данных и машинное обучение». URL: https://alexanderdyakonov.files.wordpress.com/2017/06/book_boosting_pdf. pdf (Дата обращения 03.12.2021).

4. Ким Н.Г. Прогнозирование котировок ценных бумаг методами линейной регрессии, дерева решений и с помощью многослойной нейронной сети / Н.Г. Ким, Л.Д. Хлебородова // Студент года 2021: Сборник статей Международного учебно-исследовательского конкурса в 6-ти частях, Петрозаводск, 19 мая 2021 года. - Петрозаводск: Международный центр научного партнерства «Новая Наука», 2021. - С. 288-292. - DOI 10.46916/02062021-4-978-5-00174-249-4.

5. Хлебородова Л.Д. Сравнение методов машинного обучения для задачи прогнозирования в среде Wolfram Mathematica / Л.Д. Хлебородова, Г.С. Осипов // Постулат. 2021. № 10 (72). С. 10.

6. Хлебородова Л.Д. Исследование применимости метода градиентного бустинга для решения задачи прогнозирования / Л.Д. Хлебородова // Лучшая исследовательская статья 2021: сборник статей II Международного научно-исследовательского конкурса, Петрозаводск, 01 ноября 2021 года. Петрозаводск: Международный центр научного партнерства «Новая Наука» (ИП Ивановская И.И.), 2021. - С. 278-283. - DOI 10.46916/08112021-4-978-500174-363-7.

7. Stephen Wolfram. An Elementary Introduction to the Wolfram Language. URL: https://www.wolfram.com/language/elementary-introduction/2nd-ed/ (Дата обращения 03.12.2021).

(С Л.Д. Хлебородова, 2021 